\title{
Low Temperature SEM as a Tool for Understanding Dynamic Events in Consumer Products Research, Manufacture, and Use.
}

\author{
D.S. Jacobs, ${ }^{*}$ Y.L. Boissy, ${ }^{*}$ and S.E. Lindberg* \\ *Procter \& Gamble Co., Corporate Analytical Department, Ross, Ohio 45061
}

Cryogenic freezing in-situ followed by low temperature SEM (LT-SEM) analysis is used successfully in academic studies to capture dynamic events down to the millisecond time scale [1]. Conducting such dynamic studies in the context of the consumer products industry often requires adaptation of the traditional sample preparation and analysis protocols. The modified protocols must provide results that remain scientifically sound while delivering timely insights that drive critical business decisions. We have successfully achieved this balance using LT-SEM to understand physical and chemical changes found at every stage of product development, manufacture and use. The challenges, results, and impact of this work are presented here using examples from potato dough production, silicone emulsification in liquid laundry detergents, and analgesic lotion application to skin.

The potato dough process involves blending several dry raw materials with water and oil in a mixer followed by roll-milling to create a dough sheet. Dynamic sampling of this wet dough sheet is extremely challenging since it is carried from the mill to the cutter and fryer on a belt at high speed similar to a film or papermaking process. Water content is low in these samples and is predominantly in the form of hydrates with dry ingredients. To best visualize the location of the water, slow freezing in liquid nitrogen was combined with deep etching after sample fracturing. Dough microstructure was correlated with formulation and processing changes. The results led to manufacturing efficiency improvements as well as a finished product texture upgrade (Figure 1).

Stabilizing silicones in liquid laundry detergents is difficult from both a formulation and processing perspectives. One method of stabilization is via a continuous process with a series of unit operations designed to incorporate nanoparticles at the interface of the silicone droplets. The intermediates after each unit operation are unstable on the order of minutes so shipping samples for later analysis is unacceptable. After each unit operation at the full-scale facility we used immediate, on-line analysis by light microscopy to iteratively follow silicone coalescence versus processing and formulation changes. We then used cryogenic sample freezing and LT-SEM to monitor the location of the nanoparticles within the emulsion. The result was an optimized formulation and process (Figure 2).

The application of analgesic emulsions to skin exposes the product to shear, temperature changes, and drying. These can dramatically impact microstructure, phase behavior, and performance. Invivo studies are prohibitive due to corporate policy and human subject sensibility. We studied these dynamics on cadaver skin by developing methods for freezing, cross-sectioning, and transferring samples into the LT-SEM. We visualized the loss of emulsion structure without the desired penetration into the skin (Figure 3) leading to the early cancellation of the project as a cost-savings.

\section{References:}

[1] S.A. Gallo et al., Microsc. Microanal. 5 (suppl.2) (1999) 442. 


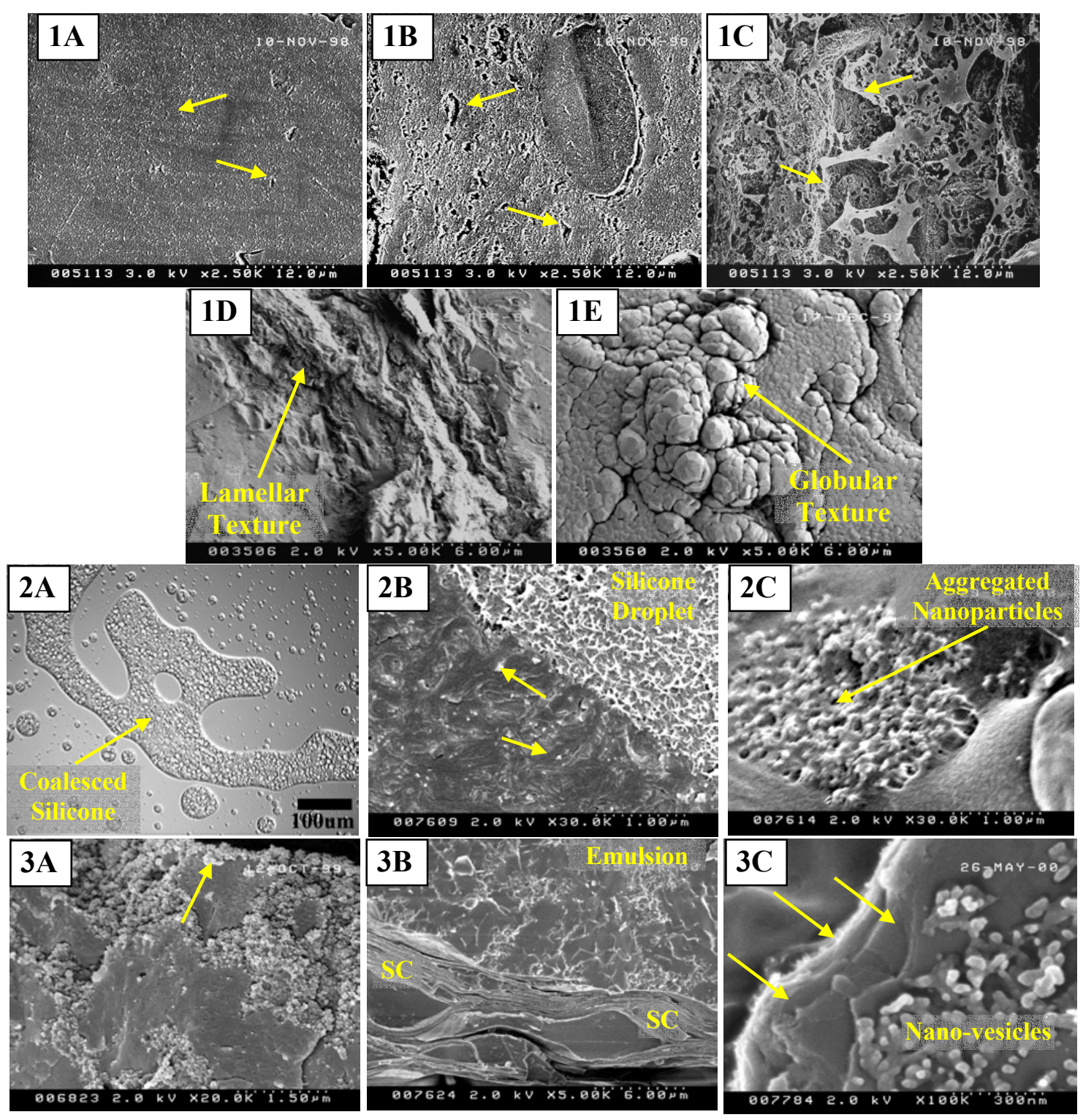

Figure 1. Heavily-etched cross-sections of potato dough. 1A) Low-moisture region with only small holes (arrows) due to etching. 1B) Medium-moisture region with larger holes (arrows). 1C) Highmoisture region with massive holes (arrows). 1D) Formula Type I with acceptable product texture. 1E) Formula Type II with improved globular product texture.

Figure 2. Liquid detergent emulsions. 2A) Light microscopy of silicone coalescence in unstable process intermediate. 2B) LT-SEM of nanoparticles (arrows) dispersed in surfactant phase of process intermediate. 2C) LT-SEM of nanoparticles aggregated within silicone phase of unstable product.

Figure 3. Cross-fractured analgesic emulsion on cadaver skin. 3A) Nano-vesicles of active ingredient migrate to air interface (arrow). 3B) No migration of nano-vesicles of active to the interface with stratum corneum layers (SC). 3C) Surface/fracture view of nano-vesicles collapsing into sheets (arrows) during drying. 